\title{
Prognostic Significance of Bax and Bcl-2 Expressions in Adenoid Cystic Carcinoma of Major and Minor Salivary Glands of Nasal and Oral Epithelium
}

\author{
Xin Xie ${ }^{1}$, Ståle Nordgård ${ }^{3}$, Ole Petter F. Clausen ${ }^{2}$ and Morten Boysen ${ }^{*}, 1$ \\ ${ }^{I}$ Department of Otolaryngology and ${ }^{2}$ The Pathology Clinic, University of Oslo, Rikshospitalet - Radiumhospitalet \\ Medical Center, Oslo, Norway \\ ${ }^{3}$ Department of Otolaryngology and Head and Neck Surgery, St. Olav University Hospital, and the Norwegian \\ University of Science and Technology, Trondheim, Norway
}

\begin{abstract}
Two apoptosis-related proteins Bax and Bcl-2 in bcl-2 gene family were studied in thirty-one randomly selected patients with T1 to T4 adenoid cystic carcinoma of the major salivary glands and minor glands of oral and nasal mucosa to investigate their prognostic value. Sections from diagnostic, formalin-fixed, paraffin-embedded specimens were stained immunohistochemically to detect Bax and Bcl-2 protein. No correlation was found between Bcl-2 and Bax expression. Bax expression correlated negatively with $\mathrm{N}$-classification $(\mathrm{p}=0.0132)$. Tumors with low Bax expression $(\mathrm{p}=0.0088)$ or high $\mathrm{Bcl}-2$ expression $(\mathrm{p}=0.0054)$ correlated with a shorter disease-free period. The combined parameters Bcl-2 - Bax and Ki-67 - Bax, showed an enhanced prognostic significance ( $p=0.002$ respectively). Our investigation revealed that Bax and Bcl-2 expression have prognostic value in ACC. The combined evaluation between these two proteins or between Ki-67 and Bax expression revealed an enhanced prognostic potential.
\end{abstract}

Keywords: Apoptosis, prognosis, treatment.

\section{INTRODUCTION}

Adenoid cystic carcinoma (ACC) is a relatively rare malignant tumor, which originate in major and minor salivary glands. The clinical behavior of this tumor is characterized by a long period of progression followed by continuing local recurrence and distant metastases over many years eventually with a fatal outcome.

Apoptosis, which involves in the deletion of cells in normal tissues as well as in malignant tumors, is a genetically predetermined mechanism that may be elicited by several molecular pathways [1-4]. Recently the proteins of bcl-2 gene family, which contain at least one Bcl-2 homology region, have been shown to play an important role in the regulation of apoptosis [5]. According to their function these proteins are divided into two groups, namely the antiand pro-apoptotic proteins. The Bcl-2, Bcl-xL and Mcl-1 are considered as antiapoptotic proteins, whereas pro-apoptotic proteins such as Bax, Bak, Bad and Bcl-xS promote apoptosis [5-8]. The mechanisms by which these proteins regulate apoptosis is largely unknown. It has been suggested that the proteins of the bcl-2 gene family heterodimerize and homodimerize with each other and that the relative proportions of these dimers determine whether or not a cell becomes apoptotic $[9,10]$. Experimental evidence indicates that proteins of the Bcl-2 family regulate apoptosis by controlling the function of mitochondria [11-14].

*Address correspondence to this author at the Department of Otolaryngology, Rikshospitalet. 0027 Oslo, Norway; Tel: + 47 95107902; Fax: + 47 23070590; E-mail: morten.boysen@rikshospitalet.no
Pro-apoptotic proteins protect mitochondria against the mitochondrial membrane permeability, presumably by binding to and neutralizing other pro-apoptotic proteins from the $\mathrm{Bcl}-2$ family, which on the contrary induce mitochondrial membrane permeabilization

Several studies have investigated the correlation between Bcl-2 expression and prognosis in ACC [15-18], but without establishing any information with regard to prognosis. The possible prognostic value of Bax expression in ACC has, to our knowledge, not been studied.

In the present study we have analyzed Bax and Bcl-2 expression in ACC in order to evaluate their possible prognostic significance. Furthermore, we tested the possible prognostic effect of a combination of these apoptotic proteins and the proliferative marker Ki-67 [19].

\section{MATERIALS AND METHODS}

The material consists of 31 randomly selected previously untreated patients with ACC admitted to the Department of Otolaryngology, Rikshospitalet, Oslo, Norway, in the period of 1983-1991. There were 15 women and 16 men ranging in age from 38 to 88 years (mean 70 years). Tumor location, TNM staging (UICC classification of 1987), treatment and follow-up has been recorded in a prospective manner. There were $17 \mathrm{~T} 1-\mathrm{T} 2$ and $14 \mathrm{~T} 3-\mathrm{T} 4$ tumors. Four patients had regional metastases at diagnosis (Table 1). None of the patients had distant metastases at diagnosis. Four of the sinonasal tumors were locally advanced $\mathrm{T} 4$ and none $\mathrm{T} 1$ or $\mathrm{T} 2$. 
Table 1. Site of the Primary Tumor According to TClassification in 31 Patients with Adenoid Cystic Carcinoma

\begin{tabular}{|c|c|c|c|c|c|}
\hline \multirow{2}{*}{ Site } & \multicolumn{4}{|c|}{ T-Classification } & \multirow{2}{*}{ Total } \\
\cline { 2 - 5 } & $\mathbf{1}$ & $\mathbf{2}$ & $\mathbf{3}$ & $\mathbf{4}$ & \\
\hline \hline Major salivary glands & 4 & 6 & 1 & 5 & 16 \\
\hline Oral cavity glands & 5 & 2 & 0 & 2 & 9 \\
\hline Sinonasal glands & 0 & 0 & 2 & 4 & 6 \\
\hline Total & 9 & 8 & 3 & 11 & 31 \\
\hline
\end{tabular}

Our treatment of ACC has, through the years been combined surgery and radiotherapy. Radical removal of tumor manifestations was attempted whenever this was possible and compatible with preservation of an acceptable cosmetic appearance and function. Three of the patients, who due to medical contraindications were unfit for surgery received radiotherapy alone. The radiation dose delivered to the primary site ranged from 60 to $70 \mathrm{~Gy}$ in once-a-day fractions of 2 Gy, 5 days a week. The four patients with $\mathrm{N}+$ status had radical neck dissection followed by radiotherapy (60 Gy) of the neck.

Since 1983 MB hold an authorization from the Norwegian Data Inspectorate to collect and evaluate data from patients admitted to our department. The local Ethical Committee and the Ministry of Health approved the study. There is no conflict of interest.

The outcome was defined as treatment failure if the primary treatment failed to eradicate the disease or if recurrence occurred locally, regionally or at distant sites $(n=11)$. Site of reccurences and survival time according to site are summarized in Table 2 . A case was censored if death resulted from an unrelated diseases or if the patient was alive with no evidence of the original tumor at the last the followup consultation/contact $(n=20)$. Complete follow-up was obtained by reviewing hospital charts, through direct contact with local hospitals, family physician or direct contact with patients or next of kin. Median follow-up for non-failures was 67(10-127) months.

Table 2. Site of Reccurences and Survival Time for 31 ACC According to Site

\begin{tabular}{|c|c|c|c|}
\hline $\begin{array}{c}\text { Primary } \\
\text { Tumor Site }\end{array}$ & $\begin{array}{c}\text { No of } \\
\text { Patients }\end{array}$ & $\begin{array}{c}\text { Site of } \\
\text { Failure }\end{array}$ & $\begin{array}{c}\text { Mean Survival } \\
\text { Time (Months) (CI) }\end{array}$ \\
\hline \hline Major salivary glands & 15 & L:1, R:0, D:5 & $87(62-113)$ \\
\hline Oral cavity glands & 10 & L:0, R:0, D:1 & $108(94-120)$ \\
\hline Sinonasal glands & 6 & L:4, R:0, D:0 & $29(7-50)$ \\
\hline Total/ overall mean & 31 & & \\
\hline
\end{tabular}

\section{Histopathology}

Successive $5 \mu \mathrm{m}$ sections were cut from formalin-fixed, paraffin-embedded pretreatment or operative specimens and mounted on gelatine coated slides. One section was stained with hematoxylin and eosin to verify the initial histopathological diagnosis, and two sections of each tumor were used for immunostaining for $\mathrm{Bax}$ and $\mathrm{Bcl}-2$ proteins respectively.

Having verified the diagnosis of ACC, histopathological sub-classification was performed based on the relative amount of the different histological subtypes (WHO). This classification distinguishes three different tumor grades. Grade 1 includes the tubular and/or cribriform patterns with no elements of solid growth pattern. Grade 2 is composed of tubular and/or cribriform patterns with less than $30 \%$ of the solid growth pattern. Grade 3 has $30 \%$ or more of the solid growth pattern [20].

\section{Immunohistochemistry}

Bcl-2 and Bax immunostaining and the evaluation were performed according to methods described previously [21]. In brief, the sections were deparaffinized by 2 washes in xylene for 5 minutes each and then dehydrated in absolute ethanol. The sections were incubated in $3 \%(\mathrm{v} / \mathrm{v})$ hydrogen peroxide in methanol (45 sec.) to block endogenous peroxidase, followed by incubation with $95 \%$ and $70 \%$ ethanol (15 sec. each), distilled water (1 min.), and phosphate-buffered saline (PBS) (5 min.). They were then heated in a pressure cooker for 5 minutes in $10 \mathrm{mM}$ citric acid buffer ( $\mathrm{pH}$ 6.0), followed by rinsing in lukewarm tap water. Thereafter the sections were then placed in Trisbuffered saline (TBS, pH 7.8) for 5 minutes, and blocked in TNK buffer (100 mM Tris 7.6 to $7.8,550 \mathrm{mM}$ sodium chloride, $10 \mathrm{mM}$ potassium chloride) which contained $2 \%$ $(\mathrm{w} / \mathrm{v})$ bovine serum albumin (BSA), $0.1 \%$ Triton X100, and $1 \%$ normal goat serum. The rabbit anti-human Bax or Bcl-2 polyclonal antibodies (Santa Cruz Biotechnology, CA, USA; 1:20 dilution of $100 \mu \mathrm{g} / \mathrm{ml}$ stock made up in TNK buffer) was added and the sections were incubated overnight in a humidified chamber at $4^{0} \mathrm{C}$. The sections were then washed once with PBS and incubated for 1 hour at room temperature in a humidified chamber with biotinylated goat anti-rabbit antibody (1:500) made up in TNK buffer, followed by washing with $\mathrm{PBS}$, incubation for 30 minutes at room temperature with streptavidin horseradish-peroxidase $(1: 20)$ made up in TNK buffer, then in a development solution containing $0.06 \%$ diaminobenzidine (DAB) and $0.1 \%(\mathrm{v} / \mathrm{v})$ hydrogen peroxide made up in TNK buffer (without goat serum, BSA, and Triton X100). The sections were finally counterstained with hematoxylin and mounted. For each batch of stained sections one positive and one negative control were included. Sections without primary antibodies were used as negative controls.

Evaluation: The sections were examined microscopically in conjunction by two of the authors (XX and OPFC) and scored according to the fraction of tumor cells stained and the staining intensity as described previously [21]. The scores for the percentages of positive tumor cells and the staining intensity were added to obtain a final score with 11 classes ranging from 0-5 (Table 3). Muscular tissue and/or normal epithelium present in the sections served as internal controls for staining intensity and were given a score of 1 . The intensity of the immunostaining was sometimes heterogeneous. Having examined the whole tumor area available in each section we classified the intensity 
according to the dominant intensity [22]. Occasional disagreements regarding the classification were discussed and a consensus reached.

Table 3. Classification Used for the Evaluation of ImmunoPhotochemical Staining of Bax and Bcl-2, in Addition, Calculation of Expression Scores

\begin{tabular}{|c|c|c|c|}
\hline Percentage of Cells Stained & Score & Staining Intensity & Score \\
\hline \hline 0 & 0 & Neg. & 0 \\
\hline $1-30$ & 1 & Weak & 0.5 \\
\hline $31-70$ & 2 & Moderate & 1 \\
\hline $71-100$ & 3 & Very intense & 2.0 \\
\hline
\end{tabular}

The scores for the percentages of cells stained and the staining intensity are added resulting in 11 classes ranging from 0 to 5 .

For all parameters, evaluated areas with pronounced inflammation, necrosis or artificial damage were avoided. The assessments were performed without knowledge concerning the clinical outcome.

The immunostaining and the evaluation of the Ki-67 expression has been presented previously [19]. The monoclonal antibody MIB1 (Immunotech, Marseille, France) was used and the results expressed as the percentage of positive nuclei among 1000 tumor cells in 400x fields evenly distributed through the largest part of the specimen.

Reproducibility: Six months after the initial evaluation eight sections were randomly selected and reevaluated for all parameters evaluated.

Statistics: The data were stored and analyzed by means of SAS 8 software (SAS Institute, Cary, NC, USA). The chisquare test was used for comparison of categorical parameters. Correlation between parameters was performed by the Pearson test. Kaplan-Maier plots and log-rank test were used to visualize and evaluate the significance of clinical and apoptosis-related variables in relation to treatment failure. A case was censored if death resulted from unrelated diseases or if the patient was alive with no evidence of the original tumor at the last follow-up consultation or contact. The DISCRIM analysis that computes various discriminant functions for classifying observations into groups on the basis of quantitative variables was used to screen possible combinations between two variables in relation to treatment failure. Prognostic significant parameters in the univariate analysis were further analyzed by means of Cox proportional hazards regression model. P-values $<0.05$ were considered statistical significance. Reproducibility was tested by means of the least squares regression analysis.

\section{RESULTS}

Bcl-2 expression was observed in all tumors, whereas Bax expression was seen in 26 of the tumors $(84 \%)$. The mean score for Bcl-2 expression was 3.5 (range from $1.5-$ 4.5), while the mean score was 2.5 (range from $0-4.5$ ) for Bax expression.

No correlation was found between Bcl-2 and Bax expression. Bax expression correlated negatively with the
N-classification $(p=0.0132)$, but not with the Tclassification. No correlation was found between Bcl-2 expression and the $\mathrm{T}$ - and $\mathrm{N}$-classifications. Ki-67 correlated neither with Bcl-2, nor with Bax expression.

Patients having tumors with Bax expression below the mean (Fig. 1) or Bcl-2 expression exceeding the mean (Fig. 2) had an unfavorable prognosis $(p=0.029$ and $p=0.024$ respectively). Table 4 summarizes the univariate analyses for clinical and immunohistochemical parameters regarding treatment failures/non-failures. Sinosasal tumours had a worse prognosis in the univariate analysis but this significance disappeared in the multivariate analysis. Ki-67 expression and $\mathrm{N}$-classification were also significant variables. Multivariate analysis revealed that Bcl-2 nodal status and Ki-67 expression were independent significant variables.

The DISCRIM analysis suggested a synergistic effect of Bcl-2 and Bax expression, and between Ki-67 expression and Bax expression in relation to treatment failures/nonfailures. According to the distribution of the plots for combined parameters in relation to treatment failures/nonfailures, we selected the mean value as cut-off levels to obtain two classes for Bcl-2 and Bax expression since the expression of these two proteins were quantified in the same way. Similarly we also selected 3-4 cut-off levels for Bax expression and Ki-67 expression, respectively, based on their distributions in order to obtain classifications for the new combined parameter Ki-67 - Bax. According to the type of prognostic association the scores for Bax expressions were subtracted from scores of Bcl-2 expression, or the score of $\mathrm{Ki}-67$ expression to obtain the new combined parameters Bcl-2 - Bax, and Ki-67 - Bax respectively. Table 5 presents the log-rank analysis for individual parameter and the combined parameters in relation to treatment failures/nonfailures. These results show that combined parameters were stronger prognostic indicators than single parameters. Figs. $(3,4)$ show the co-operative effect between Bcl-2 and Bax expressions, and between $\mathrm{Ki}-67$ and Bax expressions, respectively, in discriminating between treatment failures/non-failures.

The reproducibility of the apoptosis-related parameters was acceptable, with least square regression analysis resulting in the $\mathrm{R}^{2}$-value of 0.81 for Bax expression and 0.78 for Bcl-2 expression.

\section{DISCUSSION}

The present study shows that low Bax expression correlate with poor prognosis, whereas high expression correlated with favorable clinical outcome. To our knowledge, we demonstrate for the first time a correlation between Bax expression and prognosis in ACC. This finding is in accordance with results from previous findings in head and neck cancer [21-25]. Previous studies have reported that no correlation has been found between Bcl-2 expression and prognosis in ACC [15-18]. Results from the present study have, however, shown that high $\mathrm{Bcl}-2$ expression is associated with an unfavorable outcome. This finding is in agreement with the results on squamous cell carcinoma of the head and neck [22, 25-26] and contradict reports suggesting that there is no correlation The Different results 


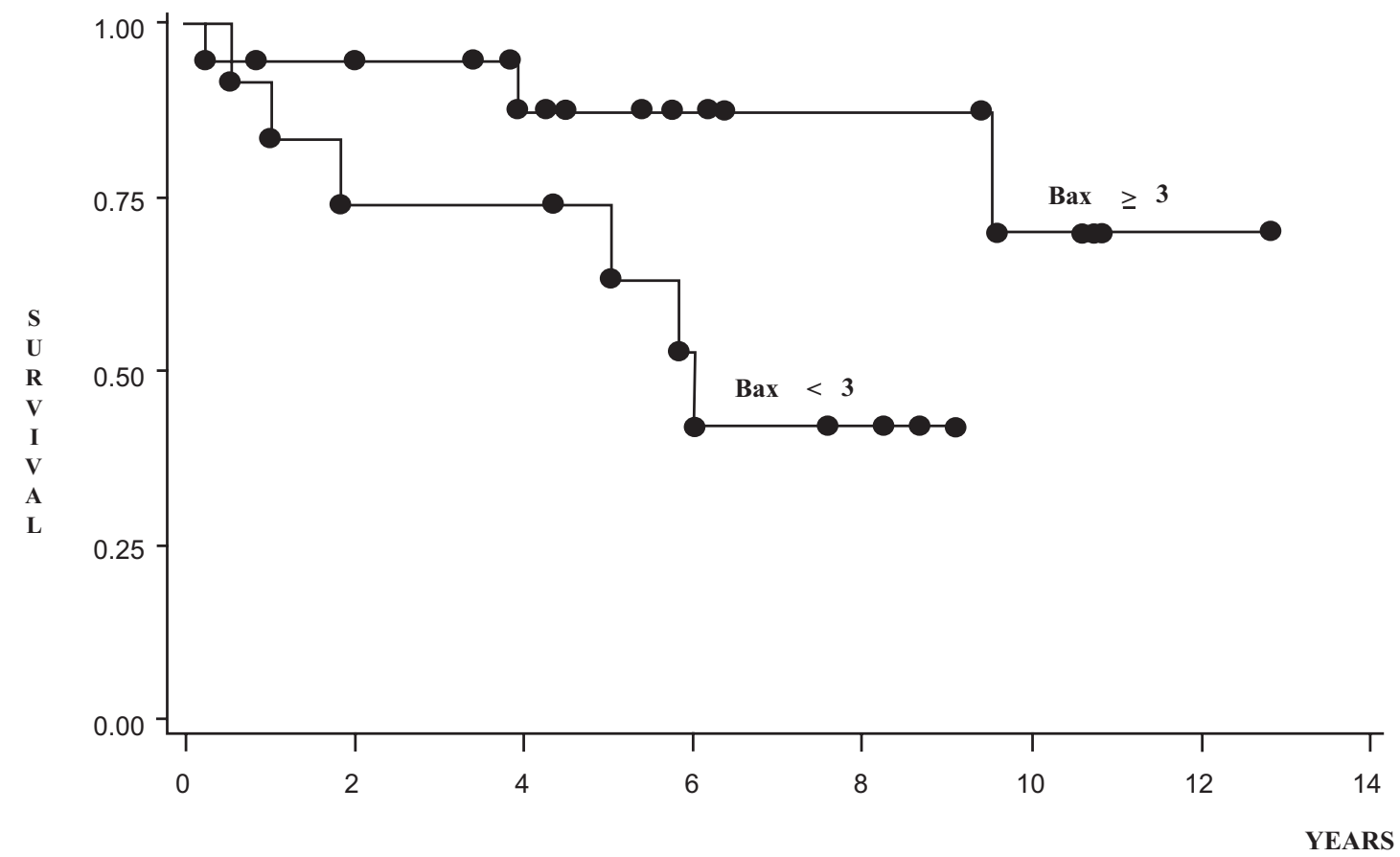

Fig. (1). Kaplan-Meier survival curves of treatment failure for Bax expression in 31 patients with adenoid cystic carcinoma ( $\mathrm{p}=0.0088$ ).

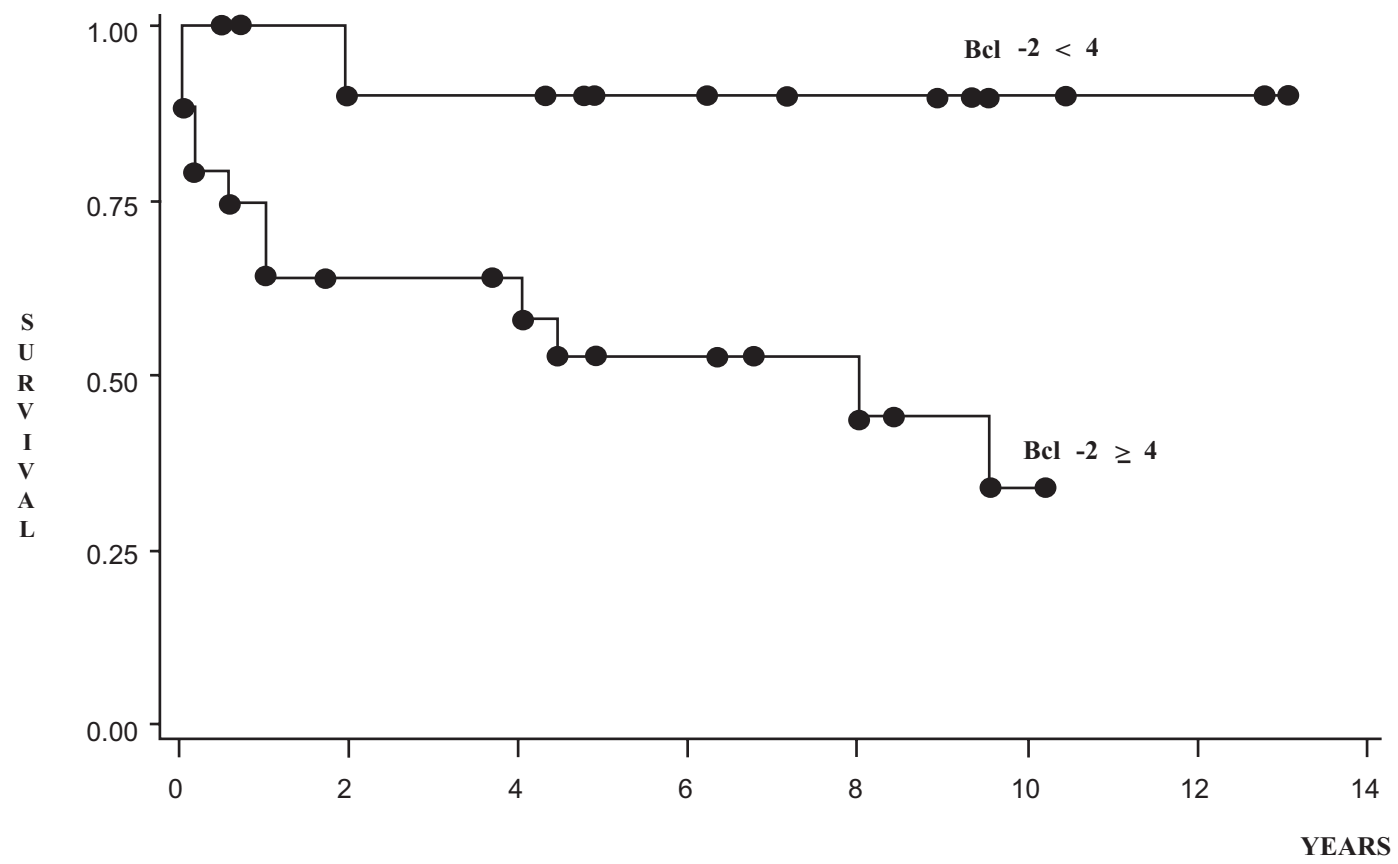

Fig. (2). Kaplan-Meier survival curves of treatment failure for $\mathrm{Bcl}-2$ expression in 31 patients with adenoid cystic carcinoma (p=0.0054).

reported on the correlation between Bcl-2 expression and prognosis, may be explained by different methods regarding evaluation of Bcl-2 expression in ACC $[15,18]$. Our method is based on both the percentages of positive tumor cells and staining intensity. This method allows a more detailed evaluation and its reproducibility is acceptable [21, 22]. In previous studies only the frequency of positive cells were evaluated, and graded in two classes, either positive or negative grading system varied markedly from only 2 classes [17] to a percentage of total tumor cells counted [18].
Additionally, the materials of previous studies were somewhat different to our study. Carlifante et al. [18] studied only the palatal tumors that have a favorable prognosis, Norberg-Spaak et al. [15] and Jia et al. [17] did not include the sinonasal carcinomas.

The lack of correlation between the apoptosis related proteins and histological grade or tumor size or stage in the present study is in accordance with findings in earlier studies where sinonasal carcinomas were omitted [15, 17-18]. 
Table 4. Log-Rank and Cox Regression Analysis for Clinical Parameters, Histological Parameter, Proliferation- and ApoptosisRelated Parameters Ki-67, Bcl-2 and Bax Expressions in Relation to Treatment for 31 Patients with ACC

\begin{tabular}{|c|c|c|c|c|c|c|}
\hline Prognostic Variables & Cut-Off Levels & Non-Failures $(n=20)$ & Failures $(n=11)$ & \multicolumn{2}{|c|}{ Log-Rank } & $\frac{\text { Cox Method }}{\text { p-Value }}$ \\
\hline T-class. & $\mathrm{T} 1-2 / \mathrm{T} 3-4$ & $12 / 8$ & $6 / 5$ & 1.15 & 0.284 & 0.775 \\
\hline N-class. & $\mathrm{N} 0 / \mathrm{N}+$ & $19 / 1$ & $8 / 3$ & 4.69 & 0.030 & 0,009 \\
\hline Histol.-class & $1 / 2 / 3$ & $12 / 5 / 3$ & $6 / 2 / 3$ & 2.93 & 0.231 & 0.877 \\
\hline KI-67 exp & $<4 \% / \geq 4 \%$ & $16 / 4$ & $6 / 5$ & 5.06 & 0.024 & 0.010 \\
\hline Bcl-2 exp. & $<4 / \geq 4$ & $11 / 9$ & $1 / 10$ & 5.12 & 0.024 & 0.007 \\
\hline Bax exp. & $<3 / \geq 3$ & $5 / 15$ & $7 / 4$ & 4.75 & 0.029 & 0.421 \\
\hline
\end{tabular}

Ns: Not significant. Exp: expression.

Table 5. Log-Rank and Cox Regression Analysis for Individual Parameters for Apoptosis-Related Variables Such as Bax and Bcl2, Proliferative Marker Ki-67, and Combined Parameter Bcl-2 - Bax, and Ki-67 - Bax in Relation to Treatment for 31 Patients with ACC

\begin{tabular}{|c|c|c|c|c|c|}
\hline Bax exp. & $<3 / \geq 3$ & $5 / 15$ & $7 / 4$ & 4.75 & 0.029 \\
\hline Bcl-2 exp. & $<4 / \geq 4$ & $11 / 9$ & $1 / 10$ & 5.12 & 0.024 \\
\hline \multicolumn{6}{|l|}{ Combined Parameters } \\
\hline Bcl-2-Bax & $\leq 0 />0$ & $19 / 1$ & $4 / 7$ & 14.02 & 0.0002 \\
\hline Ki-67-Bax & $\leq 0 />0$ & $17 / 3$ & $3 / 8$ & 13.43 & 0.0002 \\
\hline
\end{tabular}

A bewildering number of clinical (such as site of the primary tumor, $\mathrm{T}-\mathrm{N}, \mathrm{N}-$ and M-classification)and histopathological factors (histological pattern/classification, resection margins, perineuralinvasion)determine the outcome of patients with ACC. The most important feature of ACC is its unique ability to generate systematic metastases which extends over a period of 20-30 years [27, 28].

In oral tongue squamous cell carcinomas, we have demonstrated that combined evaluation of two members of the bcl-2 family, such as Bcl-2 and Bax expression, Bax and Bak expression, Mcl-1 and Bad expression, has revealed stronger prognostic potential when compared with the single variables $[22,29,30]$. In the present study, such a combined evaluation between Bcl-2 and Bax expressions has shown to enhance the prognostic significance when compared with the single parameter. Although the mechanism by which apoptosis is regulated by proteins of bcl-2 gene family is still unclear, certain domains of homology between family members, termed Bcl-2 homology domains, are critical for various aspects of their activities, including the induction or suppression of cell death. Accumulated evidence has also revealed that these proteins hetero-dimerize and homodimerize with each other and that the relative proportions of these dimers appear to determine whether a cell becomes apoptotic [9, 10]. Thus, the combined evaluation of several proteins in the bcl-2 family might be more important than changes within single protein level for the understanding of the role-played by the bcl-2 family in the regulation of apoptosis.

Furthermore, combined evaluation of the expressions of proliferative marker Ki-67 and the apoptotic marker Bax has also been shown to be a consistent stronger prognostic parameter then individual variables in the present study. This is in agreement with our previous findings in glottic and oral tongue squamous cell carcinomas [21, 31]. Total tumor progression depends on both the proliferative activity and apoptosis, and many genes reflecting different biological aspects contribute to this process. Combined evaluation of several parameters might reflect more closely the tumor development and thus has stronger prognostic significance than individual parameters.

\section{CONCLUSION}

Our investigation revealed that $\mathrm{Bax}$ and $\mathrm{Bcl}-2$ expressions have prognostic value in ACC. The combined evaluation of the expression of these two proteins, or between expressions of Ki-67 and Bax, respectively, has an enhanced prognostic potential when compared with single parameters. Because of the prolonged course of ACC, a longer observation period is needed to see if these results also cause a reduced survival. 


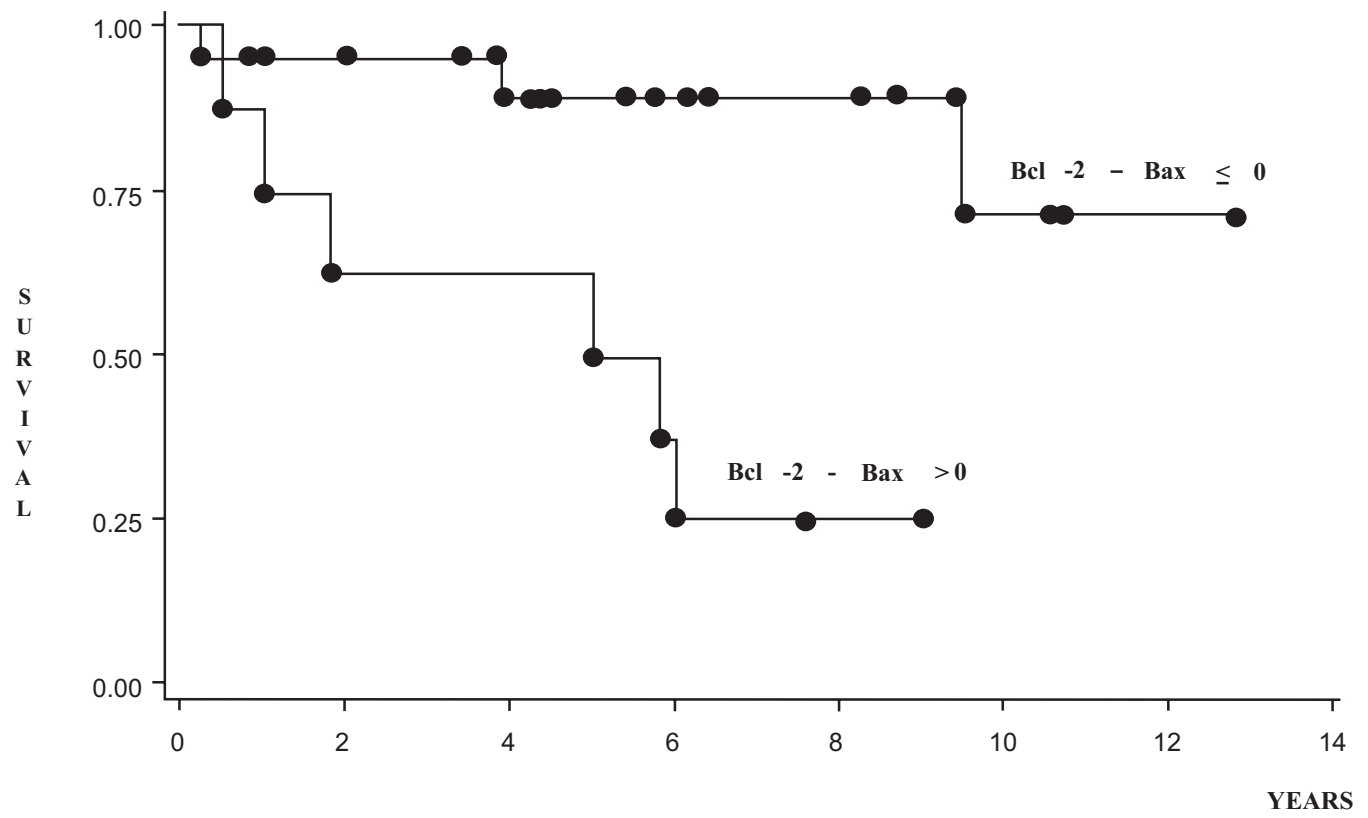

Fig. (3). Kaplan-Meier survival curves of treatment failure for Bcl-2 - Bax combination score in 31 patients with adenoid cystic carcinoma $(\mathrm{p}=0.0002)$.

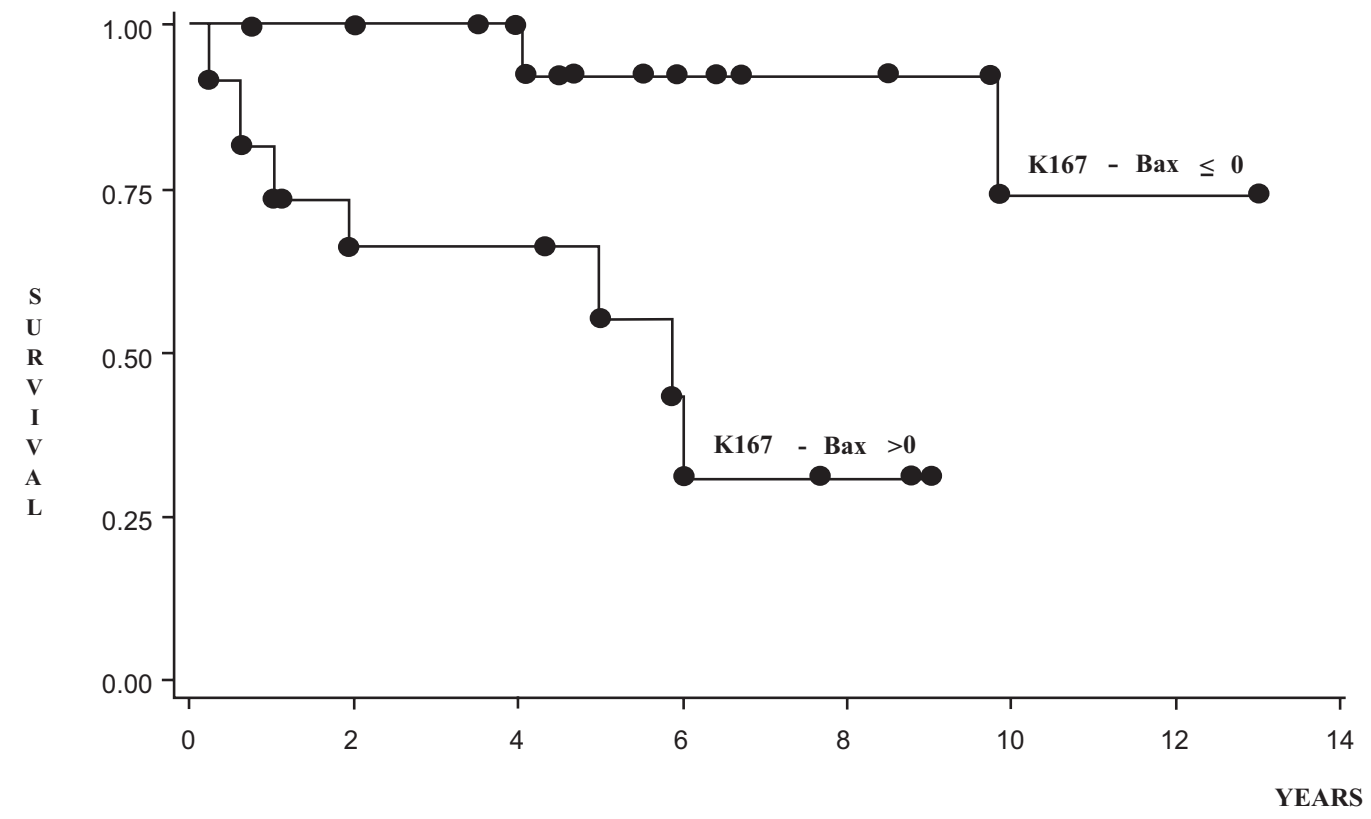

Fig. (4). Kaplan-Meier survival curves of treatment failure for Ki-67 - Bax combination score in 31 patients with adenoid cystic carcinoma $(\mathrm{p}=0.0002)$.

The evaluation of Bcl-2, Bax and Ki-67 should be made from preoperative biopsies when possible for treatment planning or from finally resected specimens to help in the decision regarding the need for radiation or for evaluating the radiation dose.

\section{ACKNOWLEDGEMENT}

This study was supported by the Norwegian Cancer Society.

\section{REFERENCES}

[1] Kerr JF, Wyllie AH, Currie AR. Apoptosis: a basic biological phenomenon with wide-ranging implications in tissue kinetics. Br J Cancer 1972; 26: 239-57.

[2] Searle J, Collins DJ, Harmon B, Kerr JF. The spontaneous occurrence of apoptosis in squamous carcinomas of the uterine cervix. Pathology 1973; 5: 163-9.

[3] Wyllie AH. The biology of cell death in tumours. Anticancer Res 1985; 5: 131-6.

[4] Scaffidi C, Fulda S, Srinivasan A, et al. Two CD95 (APO-1/Fas) signaling pathways. EMBO J 1998; 17: 1675-87. 
[5] Reed J C. Bcl-2 and the regulation of programmed cell death. J Cell Biol 1994; 124: 1-6.

[6] Hockenbery DM. Bcl-2 in cancer, development and apoptosis. J Cell Sci 1994; 18: 51-5.

[7] Boise LH, Gottschalk AR, Quintans J,Thompson CB. Bcl-2 and Bcl-2-related proteins in apoptosis regulation. Curr Top Microbiol Immunol 1995; 200: 107-21.

[8] Oltvai ZN, Milliman CL, Korsmeyer SJ. Bcl-2 heterodimerizes in vivo with a conserved homolog, Bax, that accelerates programmed cell death. Cell 1993; 74: 609-19.

[9] Oltvai ZN, Korsmeyer SJ. Checkpoints of dueling dimers foil death wishes. Cell 1994; 79: 189-92.

[10] Reed JC. Bcl-2: prevention of apoptosis as a mechanism of drug resistance. Hematol-Oncol Clin North Am 1995; 9: 451-74.

[11] Green D, Kroemer G. The central executioners of apoptosis: caspases or mitochondria? Trends Cell Biol 1998; 8: 267-71.

[12] Gross A, Jockel J, Wei MC, Korsmeyer SJ. Enforced dimerization of BAX results in its translocation, mitochondrial dysfunction and apoptosis. EMBO J 1998; 17: 3878-5.

[13] Shimizu S, Narita M, Tsujimoto Y. Bcl-2 family proteins regulate the release of apoptogenic cytochrome $\mathrm{c}$ by the mitochondrial channel VDAC. Nature 1999; 399: 483-487.

[14] Kroemer G, Reed JC. Mitochondrial control of cell death. Nat Med 2000; 6: 513-519.

[15] Norberg-Spaak L, Dardick I, Ledin T. Adenoid cystic carcinoma: use of cell proliferation, BCL-2 expression, histologic grade, and clinical stage as predictors of clinical outcome. Head Neck 2000; 22(5): 489-97.

[16] Preisegger KH, Beham A, Kopp S, Jessernigg G, Gugl A, Stammberger H. Prognostic impact of molecular analyses in adenoid cystic carcinomas of the salivary gland. Onkologie 2001; 24(3): 273-7.

[17] Jia L, Esguerra R, L, Tang X, et al. Prognostic value of apoptosis and apoptosis-associated proteins in salivary gland adenoid cystic carcinoma. Pathol Int 2004; 54(4): 217-23.

[18] Gabriele C, Mirca L, Siluano F, Bernardo B, Pellegrino C. P53, bcl-2 and Ki-67 expression in adenoid cystic carcinoma of the palate. A clinico-pathologic study of 21 cases with long-term follow-up. Pathol Res Pract 2005; 200(11-12): 791-9.
[19] Nordgard S, Franzen G, Boysen M, Halvorsen TB. Ki-67 as a prognostic marker in adenoid cystic carcinoma assessed with the monoclonal antibody MIB1 in paraffin sections. Laryngoscope 1997; 107(4): 531-6.

[20] Szanto PA, Luna MA, Tortoledo ME, White RA. Histologic grading of adenoid cystic carcinoma of the salivary glands. Cancer 1984; 54(6): 1062-9.

[21] Xie X, De Angelis P, Clausen OPF, Boysen M. Bax expression has prognostic significance that is enhanced when combined with AgNOR counts in glottic carcinomas. Br J Cancer 1998; 78: 100-5.

[22] Xie X, Clausen OPF, De Angelis P, Boysen M. Prognostic value of spontaneous apoptosis, Bax, Bcl-2, and p53 in tongue squamous cell carcinoma. Cancer 1999; 86: 913-20.

[23] Bandoh N, Hayashi T, Kishibe K, et al Prognostic value of p53 mutations, bax, and spontaneous apoptosis in maxillary sinus squamous cell carcinoma. Cancer 2002; 94(7): 1968-80.

[24] Yang YP, Qing HY, Cao Y. Correlation between prognosis and cell proliferation, apoptosis in squamous cell carcinoma of tongue. Zhonghua Kou Qiang Yi Xue Za Zhi 2003; 38(1): 31-4 (Chinese).

[25] Nix P, Cawkwell L, Patmore H, Greenman J, Stafford N. Bcl-2 expression predicts radiotherapy failure in laryngeal cancer. $\mathrm{Br} \mathrm{J}$ Cancer 2005; 92(12): 2185-9.

[26] de Vicente JC, Olay S, Lequerica-Fernandez P, Sanchez-Mayoral J, Junquera LM, Fresno MF. Expression of Bcl-2 but not Bax has a prognostic significance in tongue carcinoma. J Oral Pathol Med 2006; 35(3): 140-5.

[27] Xie X, Clausen OPF, Boysen M. Prognostic value of Bak expression in oral tongue squamous cell carcinomas. Oncol Rep 2003; 10(2): 369-374.

[28] Seifert G, Miehlke A, Chilla H. Diseases of the salivary glands. Georg Thieme Verlag 1986.

[29] Conley J, Casler JD. Adenoid cystic cancer of the head and neck. Thieme Medical Publishers Inc, 1991.

[30] Xin X, Clausen OPF, Boysen M. Prognostic value of bad and Mcl1 expression in oral tongue squamous cell carcinomas. Oncol Rep 2008.

[31] Xie X, De Angelis P, Clausen OPF, Boysen M. Prognostic significance of proliferative and apoptotic markers in oral tongue squamous cell carcinomas. Oral Oncol 1999; 35: 502-9. 